\title{
Comparison of antibacterial effects of oral rinses chlorhexidine and herbal mouth wash in patients admitted to intensive care unit
}

\author{
Gholipour Baradari A ${ }^{1}$, Darvishi Khezri H², Arabi $\mathrm{S}^{3}$
}

\author{
Mazandaran University of Medical Sciences, Tehran, Iran. hadidarvishi87@yahoo.com
}

\begin{abstract}
Background and purpose: Mouthwashes decrease the risk of VAP by reducing the number of microorganisms and their transmission and colonization in the lung. Among the oral rinses, chlorhexidine is considered as the gold standard but it has a variety of complications. The purpose of this study was to determine and compare anti-bacterial effects of the chlorhexidine gluconate and herbal mouthwashes in intensive care unit patients. Materials and methods: In this double blind randomized clinical trial, 60 patients who were admitted in ICU were divided into two equal 30 persons groups. In the first group Chlorhexidine gluconate $2 \%$ mouthwash and in the second group herbal mouth wash was used. Just before and again after 6 min of oral rinsing, salivary samples were obtained without any stimulation in order to culture Staphylococcus aureus and Streptococcus pneumoniae. The data were processed in SPSS $_{16}$ software and were analyzed by appropriate statistical tests. Results: Matrica ${ }^{\circledR}$ and chlorhexidine mouthwashes have significant antibacterial effects against Streptococcus pneumoniae and Staphylococcus aureus. Decreasing of the number of bacteria in samples after oral rinsing was significant in both groups $(p<0.001)$ but chlorhexidine was significantly more effective than herbal mouth wash in reducing the number of colonies $(p<0.001)$.

Conclusion: The herbal mouth wash has significant antibacterial effects against Staphylococcus aureus and Streptococcus pneumoniae, but its efficacy is less than chlorhexidine, so after further investigation, it would be considered as an alternative to chlorhexidine in ICUs (Tab. 3, Ref. 33). Full Text in PDF www.elis.sk.

Key words: mouth wash, chlorhexidine, Matrica ${ }^{\circledR}$, antibacterial.Listen
\end{abstract}

Pathogenic oral microflora plays an important role in several systemic diseases including cardiovascular diseases, pneumonia, endocarditis, chronic lung disease, atherosclerosis associated with endotoxin, and bacteremia (1-3). In healthy adults, streptococcus viridians is the predominant aerobic organism in the mouth flora but in ICU patients this flora often changes to Streptococcus pneumoniae and Staphylococcus aureus, that cause ventilatore associated pneumonia (VAP) (4).

Gram-positive bacterias such as Staphylococcus aureus and pneumococcus are the most common organisms causing early VAP and gram-negative agents including Pseudomonas aeruginosa causing late VAP (5). VAP begins at least 48 hours after starting mechanical ventilation with tracheal tube and is the most common nosocomial infection in ICU with an incidence rate of $9-40$ $\%$. It increases hospitalization time and costs and allocates up to $15-45 \%$ of mortality to itself (6-9). Accumulation of bacteria

${ }^{1}$ Faculty of Medicine Mazandaran University of Medical Sciences, Sari, Iran, ${ }^{2}$ Department of Nursing, Islamic Azad University, Sari Branch, Sari, Iran, ${ }^{3}$ Mazandaran University of Medical Sciences, Sari, Iran

Address for correspondence: H. Darvishi Khezri, Department of Nursing, Islamic Azad University, Sari Branch, Sari, Iran.

Gratitude: Finally, we propound our sincere gratitude to valuable efforts of the assistant for deputy of research as well as all colleagues including: Dr. Alireza Khalilian, Dr. Niloofar Jenabian, and Mr. Mohammad Ali Rezaei and Ms. Nourafarin Mirzai. in the throat is one of the most important risk factors for VAP and the relation between VAP and oral microflora is thoroughly known (10).

George and colleagues showed that 42 percent of pathogens isolated from respiratory secretions of 26 patients with VAP had previously existed in oropharynx (11). So it seems that an effective way to prevent VAP is reducing the amount of oral microorganisms.

Mouthwashes are oral irrigators that are used for oral hygien improvement. In addition, the mouthwashes are effective in reducing oral microbial flora, removing the remaining food and creating pleasant taste in the mouth. Among the mouthwashes, chlorhexidine ( $\mathrm{CHX})$ is considered as the gold standard, but has several adverse effects that are often unpleasant for the patients, such as creation of dental pigments, taste changes, mucosal irritation and dryness and injuries, squamous changes of the gums, allergies and even the occurrence of anaphylactic shock, acute respiratory distress syndrome, adverse effects on the fetus, cytotoxic effects and negative systemic effects if ingested, precipitating the gingiva to form a supragingival mass due to changes in acidity of the mouth, and also increased accumulation of bacteria after treatment with CHX (12-17). Therefore, there was always tendency to use mouthwashes that shows equality with $\mathrm{CHX}$ in terms of antimicrobial effects and at the same time has less adverse effects compared with CHX. Various researches have been done in order to find anti-microbial materials with plant origin (18). 
Among herbal mouthwashes produced domestically we can name Matrica ${ }^{\circledR}$ with trade name Camicell ${ }^{\mathrm{TM}}$, a production of Baryj Essence Company. This product contains plant extracts of chamomile flowers, with the scientific name Matricaria Chamomilla, from the Campositae family (19). Chamomile's antifungal effects particularly with regard to its effect on Candida albicans and herpes virus, and finally its anti-bacterial and anti-inflammatory effects have provided a unique position regarding the application of this mouthwash (20). Also a powerful antioxidant effects have been reported in chamomile (21).

In a study by Szalontai and colleagues in 1979 in Hungary the active antifungal effects of chamomile components were confirmed. In this study, antifungal effects of chamomile biological active ingredients including Chamazulene, Alphabisabolol, total flavonoids and Umbelliferon on fungi such as Trichophyton Mentagrophytes, Trichophyton and Candida albicans, with a concentration of 100 $\mu \mathrm{g} / \mathrm{ml}$ were confirmed (22). But less investigations have been done on its antibacterial effects against VAP producing factors.

Therefore, after more specialized tests and clinical studies, the herbal mouthwash Matrica ${ }^{\circledR}$ can be used as an alternative to chemical mouthwashes. Since the type of microorganisms in hospitalized patients is different from patients referring to dental clinics and the amount of body strength, safety and the use of oral mouthwash by these patients also differs with alert patients, and according to the problems with side effects due to synthetic antiseptic resistance $(23,24)$ we aimed to study to determine the antimicrobial effects of herbal mouthwash Matrica ${ }^{\circledR}$ in patients hospitalized in ICU.

\section{Materials and methods}

The present study was conducted to determine and compare the anti-bacterial effects of mouthwashes chlorhexidine and Matrica ${ }^{\circledR}$ in patients admitted to the intensive care unit. In our randomized double blind clinical trial study, 60 cases of patients admitted to ICU wards of Sari Imam Khomeini Educational Center were enrolled based on some inclusion criteria and were randomly divided into two groups of chlorhexidine and Matrica ${ }^{\circledR}$.

Some of the Inclusion criteria were:

1) age over 15 years and under 65 years

2) Third and fourth day of ICU stay

3) having a tracheal tube and mechanical ventilation for 3 to 4 days

4) having a nasogastric tube for 3 to 4 days

Considering that changes of oral microflora to pathogens causing VAP occur 48 to 72 hours after intubation and hospitalization in ICU (25) Thus this study was performed between the third and fourth days of hospitalization in ICU.

\section{Exclusion criteria were:}

1) re-intubation

2) a history of antibiotic treatment before hospitalization

3) pulmonary and systemic infections

4) autoimmune diseases and malignant diseases

5) history of radiation therapy and immune weakening drugs such as corticosteroids
6) history of sensitivity to mouthwash used, asthma, allergic inflammation of the nose and skin inflammation

7) Species response to oral rinse Brvz $\cdot \mathrm{hr}$

8) use of any antimicrobial mouthwash, over two months before hospitalization

9) oral mucosal lesions and advanced periodontal disease

10) negative culture samples taken before using the mouthwash of S. aureus and pneumococcal

11) coagulopathy or anticoagulant drugs

12) less than 106 colonies of bacteria in saliva samples before mouthwash

In the first group, patients received $2 \%$ oral chlorhexidine as mouthwash, manufactured by pharmaceutical company of drug city (Tehran, Iran). In the second group, herbal mouthwash Matrica ${ }^{\circledR}$ made by Baryj pharmaceutical company with a concentration of 1 to 10 (10 drops of Matrica ${ }^{\circledR}$ mouthwash in $9 \mathrm{cc}$ water) was used according to manufacturer $s$ instructions (With the recommended dose, no symptoms were observed).

The method for data collection was by interviews with patients' families, inspectation and physical examination and completion of the questionnaire. In the questionnaire, issues such as mouthwash identification code, age, sex, nutritional serum reception, drug regimen (antibiotics), presence or absence of Pneumococcus after rinsing with mouthwash, presence or absence of S. aureus after rinsing with mouthwash, total number of bacteria colonies before and after rinsing with mouthwash, history of diabetes and duration of diabetes were recorded.

Patients in both groups were matched for drug regimen (central nervous system attenuators - antibiotics - salivation modulators attenuators of the stomach $\mathrm{PH}$ ), duration of ICU hospitalization, and frequency of washing with normal saline before entering the study. In both groups all surfaces of the mouth, gums, tongues, pharynges and teeth were carefully swabbed with the mouthwash for 6 minutes until all the oral cavity surfaces were in contact with the mouthwash. Mouthwash volume in both groups was $10 \mathrm{cc}$ and at the end of washing, the solution was removed out of the mouths of patients via sterile suction. Immediately before and six minutes after the rinse, without any provocation, saliva samples were obtained for culturing S. aureus and Streptococcus pneumoniae. Time of study was the same for all patients in the morning shift, the condition of patients while doing mouthwash, in both groups was in supine position. Moreover, sterility observation, such as complete and frequent hand washes and use of sterile gloves before and after mouth wash was done.

Samples were taken using sterile swabs directly from the tonsils and postero-superior areas of oropharynx and immediately putted on the blood agar medium (made in Germany) and swab entered the liquid medium TSB for bacterial colony counting; after that, the plate with the TSB culture medium was transferred to the Microbiology Laboratory within two hours (26).

To culture $S$. aureus and pneumococcus, a blood agar medium made in Merk Company, Germany, was used. For an experimental diagnosis of Staphylococcus aureus we utilized coagulase test and Novobiocin antibiotic disk, optochin test to identify pneumococcus, and pour plate technique for counting the total colony 
number of bacteria; to determine the unit of colony counting we used CFU associated with TSB fluid media made in Quelab Company, Canada.

For counting bacteria colonies, liquid TSB medium was incubated for about 24 hours at $37^{\circ} \mathrm{C}$, then using the standard curve, the total number of bacteria was estimated. To confirm the test results, pour plate method was used in successive dilutions (20). Due to large numbers and ease of computation in statistical tests, logarithmic numbers were used.

Anti-bacterial evaluation was performed based on the presence or absence of S. aureus and pneumoncoccus in samples after the rinse, and significant differences in total colony counts between the samples before and after mouthwash were determined $(\mathrm{p}<$ 0.05 ). To compare the number of colony of bacteria t-test (Independent \& Paired) was utilized and Comparison of S. aureus and pneumococcal in a group and between two groups was done using McNemar and chi-square Tests. Data were analyzed using SPSS 15.00 software

\section{Results}

Patients in both groups in terms of sex, age, receiving TPN, history and duration of diabetes, were not significantly different (Tab. 1).

In this study it was found that chlorhexidine and Matrica ${ }^{\circledR}$ mouthwashes are effective on Strep. pneumoniae and Staph. aureus $(p=0.026$ and $p=0.001$, respectively) (Tab. 2 ).

Fisher test showed that there was a significant difference between the two groups in terms of the presence of $S$. aureus and pneumococcus after mouthwash $(p=0.02$ and $p=0.001$, respectively). Although antibacterial effects of both mouthwashes were thoroughly obvious, the mouthwash Matrica ${ }^{\circledR}$ had weaker antibacterial effect than chlorhexidine against $\mathrm{S}$. aureus and pneumococcus. Both Chlorhexidine and Matrica ${ }^{\circledR}$ mouthwashes induced significant decrease in the number of living oral bacteria colonies (Tab. 3).

The mean and standard deviation (SD) for bacteria colony numbers (log CFU) before intervention in groups chlorhexidine and Matrica ${ }^{\circledR}$ were $5.98 \pm 0.04$ and $5.97 \pm 0.04$, respectively and t-test did not show a significant difference in bacteria colony numbers before intervention; i.e. bacteria colony numbers were statistically equal before intervention $(p=0.56)$. The results showed that there was a significant difference in terms of the number of bacteria colonies in two groups after the intervention, in the chlorhexidine group, reduction of the number of colony was higher than in Matrica $\AA(t=6.10$ and $\mathrm{p}<0.001)$.

\section{Discussion}

The purpose of this study was to determine and compare the effects of anti-bacterial mouthwashes chlorhexidine and Matrica ${ }^{\circledR}$ in patients admitted to intensive care unit. The results showed that chlorhexidine and Matrica ${ }^{\circledR}$ reduced the number of bacteria colonies and were effective on S. aureus and Pneumococcus, although chlorhexidine had greater anti-bacterial effects than Matrica ${ }^{\circledR}$.
Tab. 1. Comparison of some variables between groups chlorhexidine and Matrica ${ }^{\circledR}$.

\begin{tabular}{llccc}
\hline $\begin{array}{l}\text { Group } \\
\text { Variale }\end{array}$ & & Chlorhexidine & Matrica ${ }^{\circledR}$ & $\mathrm{p}$ \\
\hline Age (years) & & $7.52 \pm 7.1$ & $7.56 \pm 8.6$ & 0.13 \\
Sex & male & $14(23.3 \%)$ & $13(21.7 \%)$ & 0.79 \\
& female & $1(26.7 \%)$ & $17(28.3 \%)$ & \\
TPN & Yes & $11(18.3 \%)$ & $16(26.7 \%)$ & 0.19 \\
& no & $19(31.7 \%)$ & $14(23.3 \%)$ & \\
Hx of Diabetes & Yes & $14(23.3 \%)$ & $13(21.7 \%)$ & 0.79 \\
& no & $16(26.7 \%)$ & $17(28.3 \%)$ & \\
Duration of & 0 & $16(26.6 \%)$ & $17(28.3 \%)$ & 0.83 \\
Diabetes & $<5 y$ & $9(15 \%)$ & $7(11.7 \%)$ & \\
& $5 y$ and more & $5(8.4 \%)$ & $6(10 \%)$ & \\
\hline
\end{tabular}

Tab. 2. Comparison of presence or absense of S. Aureus and pneumococcus before and after oral rinse.

\begin{tabular}{llccc}
\hline Group & $\begin{array}{l}\text { Patients positive } \\
\text { for }\end{array}$ & $\begin{array}{c}\text { Before } \\
\text { intervention }\end{array}$ & $\begin{array}{c}\text { After } \\
\text { intervention }\end{array}$ & $\mathrm{p}$ \\
\hline Chlorhexidine & S. aureous & $30(100 \%)$ & $7(23.3 \%)$ & 0.001 \\
& Pneumococcus & $30(100 \%)$ & $1(3.3 \%)$ & 0.001 \\
Matrica ${ }^{\circledR}$ & S. aureous & $30(100 \%)$ & $21(70 \%)$ & 0.004 \\
& Pneumococcus & $30(100 \%)$ & $8(26.6 \%)$ & 0.001 \\
\hline
\end{tabular}

Tab. 3. Comparison of colony number before and after intervention in groups chlorhexidine and Matrica ${ }^{\circledR}$.

\begin{tabular}{lccc}
\hline CFU & $\begin{array}{c}\text { Before } \\
\text { Group }\end{array}$ & $\begin{array}{c}\text { After } \\
\text { intervention }\end{array}$ & intervention \\
\hline Chlorhexidine & $5.98 \pm 0.04$ & $4.81 \pm 0.28$ & 0.001 \\
Matrica ${ }^{\circledR}$ & $5.97 \pm 0.04$ & $5.15 \pm 0.11$ & 0.001 \\
\hline
\end{tabular}

Oropharyngeal colonization is a strong independent predictive factor for later colonization of trachea and bronchi. Scannapieco and colleagues showed that the potential pathogens causing VAP, including S. aureus and Pseudomonas aeruginosa were found in the oral cavity of ICU patients (23).

Wide ranges of synthetic mouthwashes are offered in the markets of Iran of which chlorhexidine as the most effective antimicrobial mouthwash is approved by FDA and ADA. Many studies have been done about antibacterial effects of chlorhexidine mouthwash. Veksler (1998) and Arweiler (2001) demonstrated that chlorhexidine significantly reduced the number of living oral bacteria $(27,28)$. In the study Wechsler's chlorhexidine $12 \%$ was used. Although in our study chlorhexidine $2 \%$ was applied, similar results were obtained with the Wechsler's study. Maybe we can say that lesser concentrations of chlorhexidine have acceptable impact on the number of bacteria colonies. In this study, both mouthwashes had significant effects on reducing the number of oral bacteria but the effect of chlorhexidine $2 \%$ on oral bacterial count was more prominent. Vianna et al (2004), during an in-vitro evaluation of the antimicrobial activity of sodium hypochlorite and chlorhexidine, showed that chlorhexidine reduces aerobic microorganisms (S. aureus, C. albicans) in a minute or less, also chlorhexidine, in all its concentrations, eliminates all microorganisms in 30 seconds or less (29). Scannapieco (2009) after evaluating the effect of chlorhexidine on oral pathogenic bacteria in 115 trauma patients undergoing mechanical ventilation showed 
that chlorhexidine $12 \%$ reduces Staphylococcus aureus and Pseudomonas aeruginosa and Acinetobacter in dental plaques (30). In this study, oral pathogens in dental plaques which are considered as a source of growth of bacteria were evaluated, but in our study oral pathogens in the oropharyngeal area were evaluated, perhaps they are more important sources for the pathogens causing VAP in the mouths of patients under mechanical ventilation and of course this is controversial through various articles $(5,31)$. In the present study the effects of chlorhexidine $2 \%$ on S. aureus and Pneumococcus were well demonstrated.

Due to high bacterial resistance to conventional antibiotics, and relatively high costs and complications of these drugs, the use of medicinal plants has increased. Among different plants, German chamomile with scientific name of Matricaria Chamomilla has different effects form which, anti-inflammatory and antimicrobial effects are of value. Maybe herbal mouthwashes have the competency to be used as acceptable alternative for chlorhexidine in intensive care units.

Atai (2007) in an in-vitro study comparing the antifungal and antibacterial effects of mouthwashes Persica ${ }^{\mathrm{TM}}$, Matrica ${ }^{\circledR}$, and Iral wax ${ }^{\mathrm{TM}}$ with chlorhexidine showed that among these herbal mouthwashes, Matrica ${ }^{\circledR}$ has stronger antibacterial effects against Strep. Sanguis, strep. Salivarius, Strep. Sobrinus, Actinomyces Viscosus, and candida Albicans, but this difference was not statistically significant (18). Lee et al (2005) in a laboratory study showed that Chamomile extract has anti-bacterial effects on non-oral strains but they did not clarify the amount and intensity of this effect (32). Although the type of dental pathogens is different from VAP producing factors, but the study also showed significant anti-bacterial effects on agents causing early VAP. Some studies showed no effect for this plant essense against Pseudomonas aeruginosa (33) which is probably due to its resistance. In Salehi's (2005) clinical study it was found that the mouthwash Matrica ${ }^{\circledR}$ had dramatic effect in reducing microorganisms around orthodontic brackets and the results of this study are quite similar to ours, so we can express that the herbal mouthwash Matrica ${ }^{\circledR}$ not only reduces the number of bacteria colonies in healthy people but also is effective on the number of oral bacteria in patients under mechanical ventilation while they have different and more resistant flora than healthy subjects (20). Pak-nejad (2006) in a study comparing the effect of mouthwash Matrica ${ }^{\circledR}$ with chlorhexidine $2 \%$ in patients with chronic periodontitis stated that «though its effect on packet elimination (74\%) is significantly lesser than Chlorhexidine (94 $\%$ ), but this finding alone is of value and shows the impact of Matrica ${ }^{\circledR}$ on microorganisms and reducing pocket depth and perhaps still herbal compounds are not competitive enough with CHX in changing flora of microorganisms and creating a new attachment" (19). This study is the first clinical trial of mouthwash Matrica ${ }^{\circledR}$ in patients hospitalized in intensive care unit and few laboratory studies have been done about its anti-bacterial effects. In this study, using mouthwash Matrica ${ }^{\circledR}$ did not cause certain side effects, considering the short time of utilization. Chit-sazi showed that Matrica ${ }^{\circledR}$ has almost no effect on teeth discoloration (21).

Considering the antibacterial properties of herbal mouthwash Matrica ${ }^{\circledR}$ in the present and previous studies, possessing plaque and gingivitis reducing effects, and far fewer side effects than are chemical mouthwash, perhaps after further studies, especially on causes of ventilation pneumonia, antimicrobial mouthwash can be used to prevent VAP in patients hospitalized in intensive care units.

\section{References}

1. Breault LG, Fowler EB, Cuenin MF. Periodontal disease and its association with systemic disease. Mil Med 2001; 166: 85-89.

2. Marron A, Carratala J, Gonzalez-Barca E, Fernandez-Sevilla A, Alcaide F, Gudiol F. Serious complications of bacteremia caused by viridans streptococci in neutropenic patients with cancer. Clin Infect Dis 2000; 31: $1126-1130$.

3. Whitworth JM, Seymour RA. Antibiotic prophylaxis for endocarditis,prosthetic joints, and surgery. Dent Clin North Am 2002; 46: $635-651$

4. Abele-Horn M, Dauber A, Bauernfeind A. Decrease in nosocomial pneumonia in ventilated patients by selective oropharyngeal decontamination(SOD). Intensive Care Med 1997; 23: 187 - 195.

5. Augustyn B. Ventilator-Associated Pneumonia: Risk Factors and Prevention. Crit Care Nurse 2007; 27: 32 - 39.

6. Andrade D, Beraldo CC. Oral hygiene with chlorhexidine in preventing pneumonia associated with mechanical ventilation. J Bras Pneumol 2008; 34 (9): 707 - 714.

7. Mehringer L, Ibrahim EH, Prentice D, Sherman G, Schaiff R, Fraser V. Early versus late enteral feeding of mechanically ventilated patients: results of a clinical trial. JPEN J Parenter Enteral Nutr 2002; 26 (3): 174 - 181.

8. Ollendorf DA, Rello J, Oster G, Vera-Llonch M, Bellm L, Redman $\mathbf{R}$ et al. Epidemiology and outcomes of ventilator-associated pneumonia in a large US database. Chest 2002; 122 (6): 2115 - 2121.

9. Warren DK, Shukla SJ, Olsen MA, Kollef MH, Hollenbeak CS, Cox MJ et al. Outcome and attributable cost of ventilator-associated pneumonia among intensive care unit patients in a suburban medical center. Crit Care Med.2003; 31(5): 1312 - 1317.

10. Fourrier F, Duvivier B, Boutigny H, Rourrel-Delvallez M, Chopin C. Colonization of dental plaque: a source of nosocomial infections in intensive care unit patients. Crit Care Med 1998; 26: 301 - 308.

11. George DL, Falk PS, Wunderink RG, Leeper KV, Meduri GU, Steere EL et al. Epidemiology of ventilator-acquired pneumonia based on protected bronchoscopic sampling. Am J Respir Crit Care Med 1998; 158 (6): 1839 - 1847.

12. Abedipour A, Abedsaeedi J, Salehi M, Ebrahimi E. Comparison of persica and Chlorhexidine mouthwashes in prevention of stomatitis in patients receiving chemotherapy. I.J.N.R 2006; 1 (1): 41 - 46.

13. Baldo BA, Pham NH, Zhao Z. Chemistry of drug allergenicity. Curropin Allergy Chin Immunol 2001; 1: 327 - 335.

14. Ohtoshi T, Yamauchi N, Tadokoro K. IgE antibody-mediated shock reaction caused by Topical application of chlorhexidine. Clin Allergy 1986; 16: $155-161$.

15. Kurokawa A, Hirata K. Chlorhexidine gluconate ingestion resulting in fatal respiratory distress syndrome. Vet Hum Toxicol 2008; 44: 89-89.

16. Card RR, Ostda SN. Cytotoxicity and teratogenicity of chlorhexidine diacetate releasing from, hallownylon fiber. J Pharm Pharmacol 2000; 52: $772-784$. 
$556-560$

17. Dominguoz C, Hidalgo E. Mechanism underlying Chlorhexidineinduced cytotoxicity. Toxicol In vitro 2001; 15: $271-286$.

18. Atai Z, Abdollahi H, Naderipour S, Mohammadi S. Comparison of antifungal and antibacterial effects of Persica Matrica and Iralwex with Chlorhexidine mouthwashes (An in vitro study). J Dental School Shahid Beheshti University of Medical Sciences 2007; 25 ( 1).

19. Paknejad M, Jafarzadeh TS, Shamloo AM. Comparison of the efficacy of Matrica and \%0.2 Chlorhexidine mouthwashes on 3-6 mm pockets in patients with chronic periodontitis. Islamic Dental Association of Iran 18 (3): $97-92$.

20. Salehi P, Kohanteb G, Momeni Danaei Sh, Vahedi R. Comparison of the Antibacterial Effects of Persica and Matrica, Two Herbal Mouthwashes with Chlohexidine Mouthwash. Shiraz Univ Dent J 2005; 6 (1, 2): 63 - 72.

21. Chitsazi M, Shirmohammadi A, Balayi E. Effect of Herbal and Chemical Mouth-rinses on Periodontal Indices;Comparison of Matrica, Persica and Chlorhexidine. Shiraz Univ Dent J 2008; 8 (4): 54 - 60.

22. Szalontai M, Verzar-Petri G, Florian E. Data on the antifungal effect of biologically active component of Matricaria chamomilla. Acta-pharm Hang 1976; 49 (11): 232 - 247.

23. Munro CL, Grap MJ, Elswick RK, McKinney J, Sessler CN, Hummel RS. Oral Health Status and Development of Ventilator-Associated Pneumonia: A Descriptive Study. Amer Ass Crit Care Nurses 2006; 15 : $453-460$.

24. Allaker RP, Douglas CWI. Novel anti-microbial therapies for dental plaque-related diseases. Internat J Antimicrob Agents 2008; 10: 1016.

25. Randa F. Oral Care in the Intensive Care Unit. J Contemporary Dent Pract 2007; 1: 8.1.
26. Pedreira MLG, Kusahara DM, de Carvalho WB, Nunez SC, Peterlini MAS. Oral Care Interventions and Oropharyngeal Colonization in Children Receiving Mechanical Ventilation. Am J Crit Care 2009; 18 : $319-328$.

27. Arweiler NB, Netuschil L, Reich E. Alcohol-free mouthrinse solutions to reduce supragingival plaque regrowth and vitality. A controlled clinical study. J Clin Periodontol 2001; 28: 168 - 174.

28. Veksler AE, Kayrouz GA, Newman MG. Reduction of salivary bacteria by pre-procedural rinses with chlorhexidine $0.12 \%$. Crit Care Med 1998; 26 (2): $301-308$.

29. Vianna ME, Gomes BPFA, Berder VB, Zaia AA, Feraz CCR, SouzaFilho FJ. in vitro evaluation of the antimicrobial activity of chlorhexidine and sodium hypochlorite. J Oral Surg Oral Med Oral Pathol Oral Radiol Endod 2004; 97 (1): $79-84$.

30. Scannapieco FA, Yu J, Raghavendran K, Vacanti A, Owens SI, Wood K et al. A Randomized Trial of Chlorhexidine Gluconate on Oral Bacterial Pathogens in Mechanically Ventilated Patients. Crit Care 2009; 13 (4): 117.

31. Margo A, Armola HR. Effect of Oral Care on Bacterial Colonization and Ventilator-Associated Pneumonia. Am J Crit Care 2009; 18: 275 - 278.

32. Lee SS, Zhang W, Li Y. The antimicrobial potential of 14 natural herbal dentifrices: Results of an in-vitro diffusion method study. J Am Dent Assoc 2005; 136 (5): 586.

33. Owlia P, Saderi H, Rasooli I, Sefidkon F. Antimicrobial characteristics of some herbal Oils on Pseudomonas aeruginosa with special reference to their chemical compositions. Iran J Pharmaceut Res 2009; 8 (2): $107-114$. 